\title{
DETERMINATIONS OF DISTANCES TO RADIO SOURCES WITH VLBI
}

\author{
Norbert Bartel \\ Harvard-Smithsonian Center for Astrophysics
}

\begin{abstract}
The most direct method of determining distances to galactic sources outside the Solar System is the measurement of annual parallaxes. Using as an example astrometric observations of the quasars 3C345 and NRAO512 which are separated by $\sim 0.5$ at the sky, we show that under certain conditions, annual parallaxes of galactic radio sources can be determined with VLBI with an accuracy of $50 \mu$ as (standard error). This accuracy is about tenfold higher than any annual parallax accuracy ever obtained with optical astrometry.

The, so far, most direct method of determining distances to extragalactic sources combines VLBI measurements of a supernova's angular expansion velocity with optical spectroscopic measurements of the supernova's radial expansion velocity. We have estimated the distance to SN1979C in M100 in the Virgo cluster to be $20_{-6}^{+7} \mathrm{Mpc}$, a lower bound on the distance to SN1980K in NGC6946 to be $5 \mathrm{Mpc}$, and $H_{0}$ to be $65_{-20}^{+25} \mathrm{~km} \mathrm{~s}^{-1} \mathrm{Mpc}^{-1}$, with the uncertainties intended to represent standard errors.
\end{abstract}

\section{INTRODUCTION}

Determinations of distances to celestial objects have been made through the decades usually from data obtained with optical telescopes. However under certain conditions significantly more accurate determinations of such distances can be made with radio telescopes and the technique of very-long-baseline-interferometry (VLBI).

The most direct determinations of distances to celestial objects located outside the solar system have been those derived from annual parallax measurements. Optical observations of stars (Monet et al. 1987) and VLBI observations of pulsars (Gwinn et al. 1986) have yielded determinations of annual parallaxes with a standard deviation as low as $\sim 0.5$ mas. A tenfold more accurate determination of an upper bound on an annual parallax has been obtained by Bartel et al. (1986) for the quasar 3C345 relative to the quasar NRAO512. The technique used allows under certain conditions measurements of annual-parallax distances to galactic sources with unprecedented accuracy. See Reid (1988; this volume) for measurements of statistical-parallax distances.

VLBI determinations of distances, or lower bounds on them, to extragalactic sources have been obtained through supernova observations. By combining 175 
VLBI measurements of the supernova's angular expansion velocity with optical spectroscopic measurements of the supernova's radial expansion velocity, we have estimated the distance to SN1979C in M100 in the Virgo cluster, a lower bound on the distance to $\mathrm{SN} 1980 \mathrm{~K}$ in NGC6946, and $H_{0}$. We also determined the angular diameter of SN1986J in NGC891. In the remainder I discuss our measurements in more detail.

\section{GALACTIC DISTANCE DETERMINATIONS WITH ANNUAL-PARALLAX MEASUREMENTS}

\subsection{Observations of a quasar pair as a test case}

VLBI observations at nine epochs between 1971 and 1983 have been used to determine the positions of the "core" and the jet components of the quasar 3C345 $(1641+399, z=0.595)$ relative to the more distant compact quasar NRAO512 $(1638+398, z=1.67)$ which is separated from the former by $\sim 0.5$ at the sky. The observations of the 1970's and their reduction are detailed by Wittels et al. (1976) and Shapiro et al. (1979), and the observations of the 1980's and a description of the reduction of all data are given by Bartel et al. (1986). The observations had the primary result that the core of the superluminal quasar 3 C345 is stationary with respect to the quasar NRAO512, whereas the jet components of 3C345 are moving (Figure 1). Here we want to ignore the determinations of the positions of the jets and focus on those of the core only. The nine determinations of the core positions relative to those of NRAO512 are shown in Figure 2. The uncertainties ( $\sigma:$ standard deviation) in relative right ascension, $\delta \Delta \alpha$, and relative declination, $\delta \Delta \delta$, are the root-sum-squares of the corresponding standard deviations due to:

a) the ionospheric effects (only for sessions \#1-\#4 and \#7 with single-band observations)

b) the core identification uncertainties caused by the finite angular resolution and dynamic range of the quasar maps,

c) the statistical uncertainties after the latter were (uniformly) scaled for each session so that the $\chi^{2}$ per degree of freedom of the postfit residuals of the different phase delays was unity,

d) the uncertainties of the atmospheric delay,

e) the uncertainties of UT1, and

f) the uncertainties of the coordinates of the pole position, antenna sites, NRAO512 and of the values for the earth tides.

These contributions are listed for each of our observations in the 1980's in Table 1. The smallest total standard deviation was obtained for the last session, $39 \mu$ as in $\Delta \alpha$ and $49 \mu$ as in $\Delta \delta$, with the uncertainty of locating the core in the brightness distribution of $3 \mathrm{C} 345$ dominating the error budget. This uncertainty was $35 \mu$ as in both coordinates which is equal to $~ 7 \%$ of the FWHM of the Gaussian restoring beam for our high-frequency map. Any further improvement in the accuracy of position determination of any source could most likely only come from a decrease of this dominant error, either by improving the dynamic range of the map or, more effectively, by increasing the angular resolution of the VLBI array. 


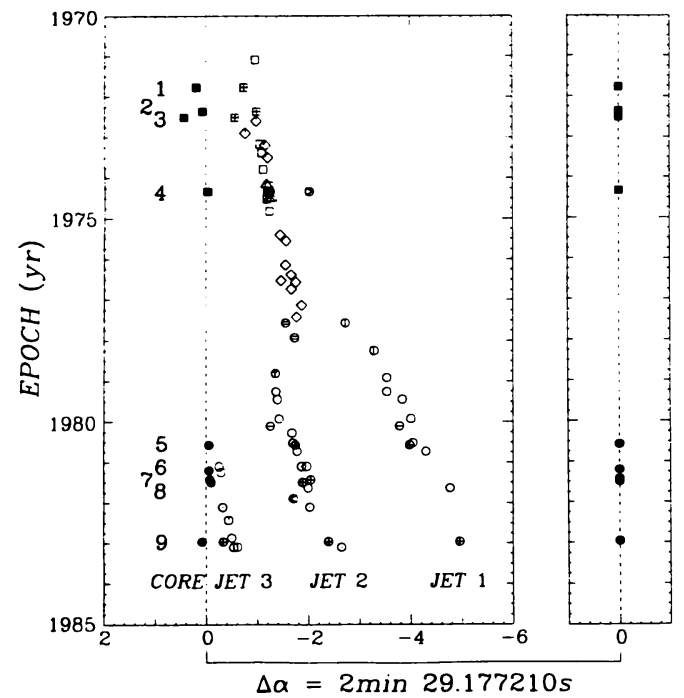

Relative RA (mas)
Figure 1. The differences in right ascension ( $\mathrm{J} 2000$ ) between the core component of $3 \mathrm{C} 345$ and the single compact component of NRAO512 for the less accurate observations in the 1970's ( $\square, \# 1-\# 4)$ and for the more accurate observations in the 1980's ( $\#$, $\#$-9). The dashed line marks the weighted mean of these differences. Other symbols indicate the positions of the jet components of $3 \mathrm{C} 345$.
RELATIVE CORE POSITIONS

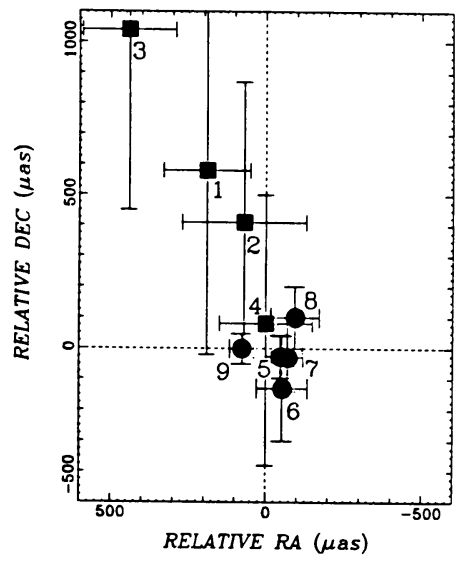

Figure 2. The positions of the core component of $3 \mathrm{C} 345$ relative to the position of the single component of NRAO512. The origin of the diagram lies at the weighted mean of the relative position estimates.
RELATIVE CORE POSITIONS

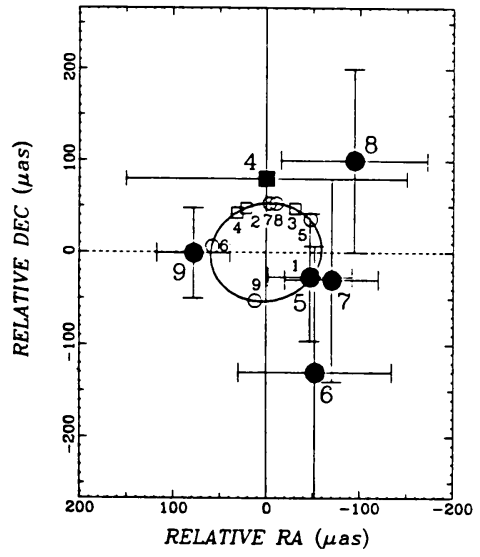

Figure 3. Enlarged inner portion of Figure 2. The ellipse indicates the predicted apparent motion of the core of $3 \mathrm{C} 345$, and the light symbols the core's predicted apparent positions at epochs, for the upper bound on any annual parallax of $60 \mu$ as. 
We estimated the parallax, $\pi$, along with the relative right ascension, $\Delta \alpha$, and declination, $\Delta \delta$, at the epoch of our first observations, and the proper motion components, $\mu_{\alpha}, \mu_{\delta}$, by a weighted-least-squares fit to our position determinations.

Table 1

Error Budget for Observations in the 1980's of 3 C345 and NRAO512

\begin{tabular}{|c|c|c|c|c|c|c|c|c|c|}
\hline \multirow[t]{3}{*}{$\#$} & \multirow[t]{3}{*}{ Epoch } & \multirow{2}{*}{$\begin{array}{l}\text { Observ. } \\
\text { freq. }\end{array}$} & \multirow{2}{*}{$\begin{array}{l}\text { Ionosph. } \\
\text { effect at } \\
8.4 \mathrm{GHz}^{1}\end{array}$} & \multicolumn{5}{|c|}{ CContributions due to: ${ }^{2}$} & \multirow{3}{*}{$\begin{array}{l}\text { Total } \\
(a-f)\end{array}$} \\
\hline & & & & a) $)^{3}$ & b) & c) & d) & $e)^{5}$ & \\
\hline & & $(\mathrm{GHz})$ & \multicolumn{6}{|c|}{$\left.\begin{array}{r}\delta \Delta \alpha \\
-(\mu \mathrm{as}\end{array}\right)$} & \\
\hline 5 & 1980.57 & $2.3 / 8.4$ & 6,50 & - & 35,35 & 28,53 & 7,18 & 3,21 & 45,69 \\
\hline 6 & 1981.20 & $2.3 / 8.4$ & 12,10 & - & 70,70 & 16,34 & 38,121 & 10,82 & 82,170 \\
\hline 7 & 1981.43 & 10.7 & - & 30,30 & 35,35 & 8,17 & 14,43 & 12,82 & 50,110 \\
\hline 8 & 1981.45 & $23 . / 8.4$ & 67,68 & - & 70,70 & 35,66 & 8,25 & 1,17 & 78,100 \\
\hline 9 & 1982.96 & $2.3 / 8.4$ & 83,49 & - & 35,35 & 15,22 & 8,19 & 3,18 & 39,49 \\
\hline
\end{tabular}

1 The magnitudes of the differences between the values for the positions determined from the $8.4 \mathrm{GHz}$ data only and the (largely ionosphere free) $2.3 / 8.4 \mathrm{GHz}$ data. The magnitudes indicate the effect of the ionosphere at $8.4 \mathrm{GHz}$ and were used to estimate the ionospheric effect a) on our astrometric result obtained for epoch 1981.43 from single-band data only.

2 Contributions due to f) are smaller than $3 \mu$ as in $\Delta \alpha$ and $10 \mu$ as in $\Delta \delta$.

3 Dashes indicate that $2.3 / 8.4 \mathrm{GHz}$ data were used for the analysis. These data are largely free from ionospheric effects. We, however, caution that any mismatch of the fiducial points in the $2.3 \mathrm{GHz}$ and $8.4 \mathrm{GHz}$ maps is carried over to the final position determination at the $7 \%$ level.

4 The uncertainties of the atmospheric zenith delays were assumed to be $0.3 \mu \mathrm{s}$, except for stations where meteorological data were recorded, or where such delays could be estimated from geodetic VLBI observations immediately preceding or following our astrometric observations. In the latter cases we assumed uncertainties of $0.1 \mu \mathrm{s}$.

5 The uncertainties of UT1 were assumed to be $0.5 \mathrm{~ms}$, except for sessions \#5, $\# 8$, and \#9 for which UT1 could be estimated from above mentioned geodetic VLBI observations. For these sessions we assumed uncertainties of $0.1 \mathrm{~ms}$.

The result is $\pi=10 \pm 50 \mu$ as, with the uncertainty denoting $1 \sigma$, scaled so that it corresponds to $\chi^{2}$ per degree of freedom of unity. Figure 3 shows an enlarged portion of the inner part of Figure 2 with the ellipse describing the expected apparent motion of the core of 3C345 due to an annual parallax of $60 \mu \mathrm{as}$ (upper bound of above determination).

From the upper bound on the relative annual parallax of $3 \mathrm{C} 345$ and NRAO512, we can place a lower bound on the distance to 3 C345 of $15 \mathrm{kpc}$ (85\% 
confidence limit), if NRAO512 is at a sufficiently greater distance from Earth, i.e. if NRAO512 can be regarded as a background source. This bound is, by far, the largest yet obtained for an annual-parallax distance and with the above assumption, would place the quasar outside the Galaxy.

\subsection{Possible observations of pairs of galactic sources and quasars}

Instead of promoting the usefulness of determinations of annual parallaxes, or upper bounds on them, for more extragalactic sources, we use our result as an example for the accuracy that could be obtained for the determination of annual parallaxes of galactic sources. Prime candidates for annual-parallax determinations are radio pulsars and radio stars, the latter being, e.g., RS CVn systems, x-ray emitters, and Mira variables (see Lestrade 1988 for a description of radio stars). If, e.g., for a galactic radio source six position determinations over the course of a year are made with only $1 / 4$ of the accuracy we obtained for the last determination of the position of $3 \mathrm{C} 345$ relative to that of NRAO512, then annual-parallax distances can be determined with an accuracy of $\sim 10 \%$ for sources up to $1 \mathrm{kpc}$ away from Earth.

\section{EXTRAGALACTIC DISTANCE DETERMINATIONS WITH SUPERNOVA OBSERVATIONS}

\subsection{SN1979C}

The supernova SN1979C in the galaxy M100 [NGC4321, distance $=11.8 \mathrm{Mpc}$ (de Vaucouleurs 1982) and 22.5 Mpc (Sandage and Tammann 1985)] in the Virgo cluster has been emitting radio radiation; at $5 \mathrm{GHz}$ the flux-density maximum of $\sim 7 \mathrm{mJy}$ occurred about one year after the explosion which we assume to have happened on 1979 April 1. The flux density has decreased since the time of the maximum by $\sim 50 \%$ (Weiler et al. 1986). Figure 4 displays a radio map of the galaxy with the supernova located in one of the galaxy's spiral arms.

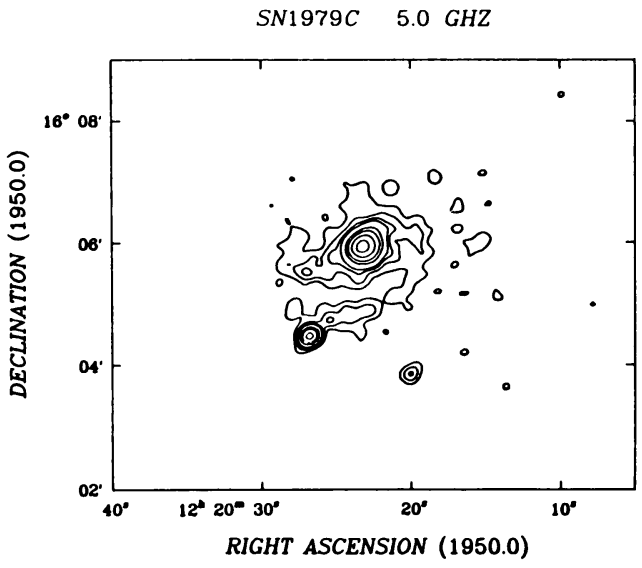

Figure 4. Radio map of SN1979C, located at the southern edge of a spiral arm of the galaxy, M100. The map was made with the VLA on 1982 Dec 8. 
A VLBI determination, at time $t$ after the explosion, of the angular size, $\Theta$, of a supernova's radiosphere, coupled with an optical-spectroscopic determination of the radial expansion velocity $v_{\mathrm{ph}}$, at time $t_{\mathrm{ph}}$ after the explosion, of the supernova's photosphere allows estimates to be made of the distance, $D$, to the supernova's host galaxy and of Hubble's constant (Bartel 1985, Bartel et al. 1985, see also Bartel 1986, for a comparison of this method with other methods that use supernovae as distance indicators):

$$
\begin{aligned}
D & =\frac{2 \mu \eta v_{\mathrm{ph}} t}{\kappa \Theta m}\left[\frac{t}{t_{\mathrm{ph}}}\right]^{m-1} \\
H_{0} & =\frac{v_{\text {redshift }}+v_{\text {infall }}}{D},
\end{aligned}
$$

The parameter $\mu$ describes the effect of any deviation from spherical symmetry on the distance determination, $\eta$ the ratio between the expansion velocity of the radiosphere and that of the photosphere, $m$ a measure of the deceleration (or acceleration) of $\Theta$, with $\Theta \alpha t^{m}$, and $\kappa$ the model dependence of $\Theta$. We define the parameter $\kappa$ so that $\kappa=1.0$ for a supernova having a shell-like brightness distribution with a shell thickness of $\sim 15 \%$. The parameter $\kappa$ ranges between $\sim 1.3$ for the brightness distribution being a uniform sphere and $\sim 0.8$ for the distribution being a ring (see, e.g., Marscher 1985). For comparison, $\kappa=0.7$, for the FWHM of a Gaussian. The parameter $v_{\text {redshift }}$ denotes the redshift velocity of the host galaxy, and $v_{\text {infall }}$ its correction due to local peculiar velocities. For a uniformly expanding, spherically symmetric supernova shell equation (1) becomes simply: $D=\frac{2 \eta v_{\mathrm{ph}} t}{\Theta}$.

The goal is to determine observationally each of the parameters in eq. (1) and thereby to estimate a host galaxy's distance and $H_{0}$. We made VLBI observations of SN1979C at several epochs with an array of usually four telescopes, three located in the US (NRAO, OVRO, VLA) and one in Europe (Bonn). The measured visibility amplitudes at an observing frequency of $5 \mathrm{GHz}$ and the predictions from a fit of a uniform sphere model to the amplitudes are given in Figure 5. The corresponding angular-diameter determinations for $\kappa=1.0$ are displayed in Figure 6 , with each of the uncertainties denoting the root-sum-square of the statistical standard error and a contribution due to antenna gain uncertainties. This contribution was taken as the standard error of the diameter estimates obtained by changing, in turn, the sensitivities of each antenna of the array by $\pm 10 \%$ while keeping the sensitivities of the other antennas fixed.

From the six dependent parameters in eq. (1), two have so far been determined with VLBI: $\Theta$ and $m$. From a weighted least-squares fit to the diameter determinations we estimated: $\Theta=0.83 \pm 0.05$ mas for the time of our first VLBI observations $3.69 \mathrm{yr}$ after the supernova explosion, and $m=1.03 \pm 0.15$. One other parameter, $v_{\mathrm{ph}}$, is known from optical spectroscopic observations (Branch et al. 1981): $v_{\mathrm{ph}} \sim 10000 \mathrm{~km} \mathrm{~s}^{-1}$, for $t_{\mathrm{ph}}=0.6 \mathrm{yr}$, after the explosion.

Since the remaining three parameters have not yet been determined, we assume reasonable values and standard errors for them.

Based on the assumption that the radio emission emanates from the shock front region that expands with the largest velocity, and the observation that the largest expansion velocity of SN1979C was $\sim 12000 \mathrm{~km} \mathrm{~s}^{-1}$ (Branch et al. 1981), we adopt a value for $\eta$, so that $\eta v_{\mathrm{ph}}=11000 \pm 1000 \mathrm{~km} \mathrm{~s}^{-1}$. The assumed value 

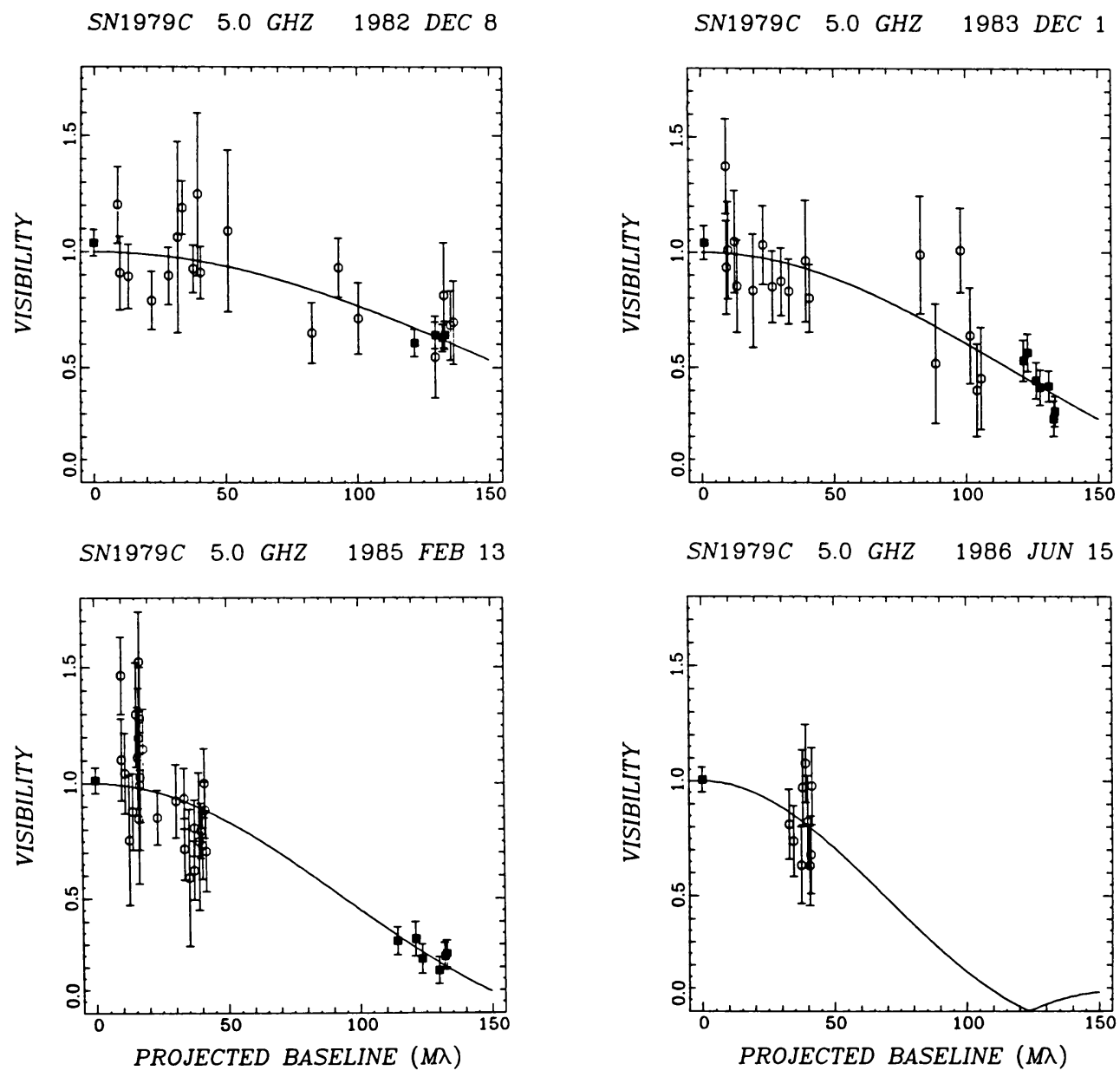

Figure 5. Measured visibility amplitudes at $5.0 \mathrm{GHz}$ and predictions from the fit of a uniform sphere model. The filled squares show the most significant data points, obtained with the VLA alone and with the Bonn-VLA interferometer.

for $\eta$ is consistent with the results obtained from VLBI observations of SN1987A in the Large Magellanic Cloud (Shapiro et al. 1988). Based on observations that many SNRs are approximately circularly shaped (Green 1984), we adopt $\mu=1.0 \pm 0.15$. Based on observations that $\sim 90 \%$ of all known SNRs with discernible morphology have a shell-like structure (Green 1984), we adopt $\kappa=1.0 \pm 0.15$.

With these values and uncertainties we get:

$$
D=20_{-6}^{+7} \mathrm{Mpc} \text {. }
$$

If we further take $v_{0}+v_{\text {infall }}=1250 \pm 150 \mathrm{~km} \mathrm{~s}^{-1}$ for the Virgo cluster center (see 12 references in Bartel et al. 1985) and assume that the distance to M100 is within 
THE EXPANSION OF SN1979C

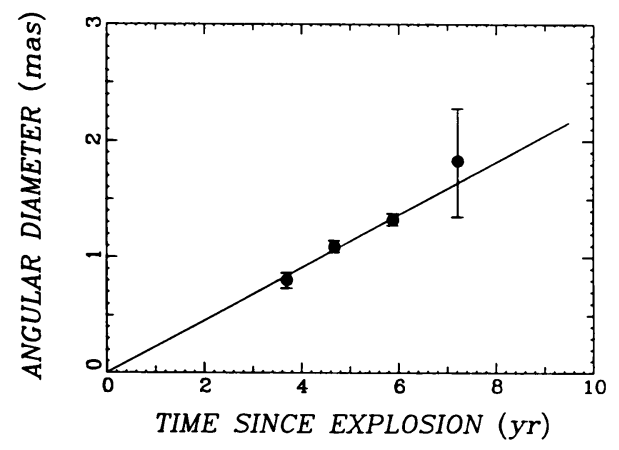

Figure 6. The angular diameter determinations for a shell model. For the extreme models of a ring and a uniform sphere, the ordinate scale has to be multiplied by 0.8 and 1.3 , respectively. The solid line represents uniform expansion $(m=1)$, which is consistent with our weighted least-squares solution for $m$.

$10 \%$ of the distance to this center, since M100 is within $5^{\circ}$ of the center and has a redshift consistent with this location, we get

$$
H_{0}=65_{-20}^{+25} \mathrm{~km} \mathrm{~s}^{-1} \mathrm{Mpc} \text {, }
$$

with the uncertainties of both estimates intended to represent standard errors. A more complete error analysis is pending. The accuracy of these updated estimates is somewhat higher than the accuracy of our earlier estimates (Bartel et al. 1985) mostly because more data have been obtained and analyzed since then.

\section{$3.2 \mathrm{SN} 1980 \mathrm{~K}$}

The supernova SN1980K in the galaxy NGC6946 [distance $=7.1 \mathrm{Mpc}$ (de Vaucouleurs 1979) and 10.5 Mpc (Sandage and Tammann 1974)] emitted radio radiation of $\sim 2.5 \mathrm{mJy}$, at $5 \mathrm{GHz} 100$ days after the explosion, and has since then also weakened considerably.

We conducted VLBI observations of SN1980K at $2.3 \mathrm{GHz}$ on 1983 May 7 with the sensitive NASA 64-m antennas at Goldstone, CA and Madrid, Spain. The supernova was detected in two out of three adjacent 13-min scans. The visibility amplitudes determined in the two scans, and an upper bound determined in the third scan, are shown in Figure 7. Since the visibility amplitudes of the three segments are expected to be approximately Gaussian distributed, we can compute their mean value and standard error and infer that SN1980K was unresolved at the epoch of our VLBI observations, $2.54 \mathrm{yr}$ after the assumed date of explosion on 1980 Oct. 17. The formal value for the diameter of a shell model is: $\Theta=1 \pm 1$ mas, equivalent to $\Theta<2$ mas. The prediction from this model is also shown in Figure 7 .

We computed a lower bound on the distance to SN1980K and its host galaxy by again using eq. (1). The last optical spectroscopic observation made $1.82 \mathrm{yr}$ after the explosion of the supernova, indicates $v_{\mathrm{ph}} \sim 7900 \mathrm{~km} \mathrm{~s} \mathrm{~s}^{-1}$ (Uomoto and Kirshner 1986). We take $\eta v_{\mathrm{ph}}=9500 \pm 1000 \mathrm{~km} \mathrm{~s}^{-1}$, consistent with the supernova's maximum expansion velocity of $\sim 9500 \mathrm{~km} \mathrm{~s}^{-1}$ about one month after the explosion. Assuming the remaining parameters in eq. (1) to 


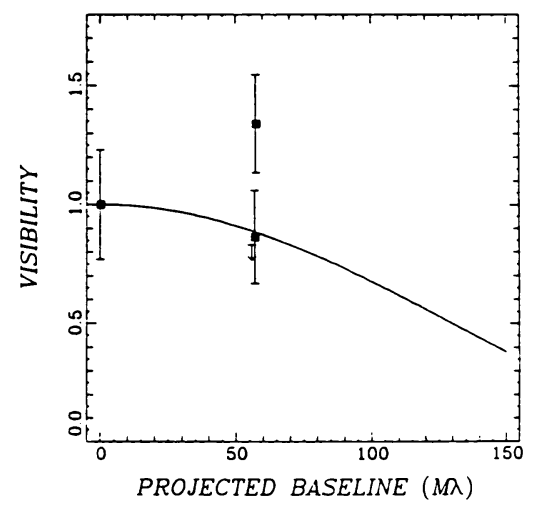

Figure 7. Visibility amplitudes and the prediction from a uniform sphere model. The visibility amplitude at zero spacing was obtained from interpolating between several measurements made with the VLA by Weiler et al. (1986) at 1.4 and $5 \mathrm{GHz}$ two days to seven weeks prior to our VLBI observations.

be: $m=1.0 \pm 0.15, \mu=1.0 \pm 0.15$ and $\kappa=1.0 \pm 0.15$, we formally get: $D=10_{-5}^{+\infty} \mathrm{Mpc}$, equivalent to

$$
D>5 \mathrm{Mpc} \text {, }
$$

with the uncertainties being again intended to represent standard errors.

\subsection{SN1986J}

The supernova SN1986J in the galaxy NGC891, at 0.56 the distance to the center of Virgo (Aaronson et al. 1982), has been by far the strongest radio supernova detected to date. The flux density reached $128 \mathrm{mJy}$ at $5 \mathrm{GHz}$ on 1986 May 1 (Rupen et al. 1987). Optical spectroscopy revealed an extremely small expansion velocity of $v_{\mathrm{ph}} \sim 1000 \mathrm{~km} \mathrm{~s}^{-1}$ (Rupen et al. 1987).

We conducted VLBI observations of SN1986J at $1.7 \mathrm{GHz}$ on $1986 \mathrm{Sept}$. 29 (Bartel et al. 1987) and at $10.7 \mathrm{GHz}$ on $1987 \mathrm{Feb} .23$, and determined the supernova's angular sizes for $\kappa=1: 2.0 \pm 0.3$ mas and $1.4 \pm 0.1$ mas, respectively, with the former diameter at $1.7 \mathrm{GHz}$ being most likely broadened appreciably by scattering in the interstellar medium.

Unfortunately for our ability to estimate the supernova's distance, the supernova's date of explosion has not yet been well determined. Since the supernova remained undetected till at least 1975 (Allen et al. 1978) and was first detected in 1984 (van der Hulst et al. private communication with Rupen et al. 1987), the supernova probably exploded sometime in between. Further, optical spectroscopic observations commenced only after the discovery of the supernova at radio wavelengths in 1986 . Crucial data from the early expansion of the supernova were therefore never obtained. However, if we assume for SN1986J the same values and uncertainties for the parameters $m, \eta, \mu$ and $\kappa$, we determined or adopted for SN1979C, then our value for the ratio between the distances to SN1986J and Virgo is at least $40 \%$ smaller than that of Aaronson et al. (1982). More VLBI and optical spectroscopic observations are needed to determine accurately the supernova's expansion velocity and morphology and to shed more light on this rather unusual source. 


\section{CONCLUSIONS}

Astrometry with VLBI has enabled us to determine an upper bound on the annual parallax of the quasar 3C345 relative to the quasar NRAO512 with a standard error of $50 \mu$ as. Such accuracy is sufficient for the determination of annual-parallax distances to galactic compact radio sources up to $\sim 1 \mathrm{kpc}$ away from Earth with an uncertainty smaller than $10 \%$.

Distances to extragalactic sources and Hubble's constant can be determined by using a combination of VLBI and optical spectroscopy of supernovae. This combination represents the, so far, most direct method of such determinations and should allow, under certain conditions, measurements of distances to galaxies up to $40 \mathrm{Mpc}$ away from Earth with an uncertainty smaller than $20 \%$.

This work was supported by the NSF under grant no. AST 85-19763.

\section{REFERENCES}

Aaronson, M. et al. 1982, Ap. J. Suppl., 50, 241.

Allen, R. J., Baldwin, J. E. and Sancisi, R. 1978, Astr. Ap., 62, 397.

Bartel, N. 1985, in Supernovae as Distance Indicators, Lecture Notes in Physics, ed. N. Bartel (Springer-Verlag, Berlin), 224, 107.

Bartel, N. 1986, in Highlights of Astronomy, ed. J. P. Swings, 7, 655.

Bartel, N., Herring, T. A., Ratner, M. I., Shapiro, I. I., and Corey, B. E. 1986, Nature, 319, 733.

Bartel, N., Rogers, A. E. E., Shapiro, I. I., Gorenstein, M. V., Gwinn, C. R., Marcaide, J. M., and Weiler, R. W. 1985, Nature, 318, 25.

Bartel, N., Rupen, M., and Shapiro, I. 1987, IAU Circ. 4292.

Branch, D., Falk, S. W., McCall, M. L., Rybski, P., Uomoto, A., and Wills, B. J. 1981, Ap. J., 244, 780.

de Vaucouleurs, G. 1979, Ap. J., 227, 380.

de Vaucouleurs, G. 1982, Ap. J., 253, 520.

Green, D. A. 1984, M. N. R. A. S., 209, 449.

Gwinn, C. R., Taylor, J. H., Weisberg, J. M., and Rawley, L. A. 1986, A. J., 91, 338.

Lestrade, J.- F. 1988, this volume.

Marscher, A. P. 1985, in Supernovae as Distance Indicators, Lecture Notes in Physics, ed. N. Bartel (Springer-Verlag, Berlin), 224, 130.

Monet, D. G., Dahn, C. C., Harris, H. C., Ables, H. D., Luginbuhl, C. B., Pier, J. R., and Vrba, F. J. 1987, Bull. AAS, 19, 79.09.

Reid, M. J. 1988, this volume.

Rupen, M. P., van Gorkom, J. H., Knapp, G. R., Gunn, J. E., and Schneider, D. P. 1987 A. J., 94, 61.

Sandage, A., and Tammann, G. H. 1974, Ap. J., 194, 559.

Sandage, A., and Tammann, G. H. 1985, in Supernovae as Distance Indicators, Lecture Notes in Physics, ed. N. Bartel (Springer-Verlag, Berlin), 224, 1.

Shapiro, I. I. et al. 1979, A. J., 84, 1459.

Shapiro, I. I. et al. 1988, this volume.

Uomoto, A., and Kirshner, R. P. 1986, Ap. J., 308, 685.

Weiler, K. W., Sramek, R. A., Panagia, N., van der Hulst, J. M., and Salvati, M. 1986, Ap. J., 301, 790.

Wittels, J. J. et al. 1976, A. J., 81, 933. 